\title{
A reevaluation of erythroid predominance in Acute Myeloid Leukemia using the updated WHO 2016 Criteria
}

\author{
Elizabeth Margolskee $\mathbb{D}^{1} \cdot \mathrm{Geoff}^{\mathrm{Mikita}}{ }^{1} \cdot$ Bryan $\mathrm{Rea}^{2} \cdot$ Adam Bagg $^{2} \cdot$ Zhuang Zuo $^{3} \cdot$ Yi Sun $^{3} \cdot$ Maitrayee Goswami $^{3}$. \\ Sa A. Wang ${ }^{3}$. Jean Oak ${ }^{4}$. Daniel A. Arber ${ }^{4}$ M. Brandon Allen ${ }^{5}$. Tracy I. George ${ }^{5}$. Heesun J Rogers ${ }^{6} \cdot$ Eric Hsi $^{6}$. \\ Robert P. Hasserjian ${ }^{7} \cdot$ Attilio Orazi $^{1}$
}

Received: 23 August 2017 / Revised: 1 November 2017 / Accepted: 23 November 2017 / Published online: 5 February 2018

(c) United States \& Canadian Academy of Pathology 2018

\begin{abstract}
The 2016 WHO update changed the diagnostic criteria for myeloid neoplasms with erythroid predominance, limiting the diagnosis of acute myeloid leukemia to cases with $\geq 20 \%$ blasts in the bone marrow or peripheral blood. Although acute myeloid leukemia with $\geq 50 \%$ erythroid cells has historically been presumed to represent acute myeloid leukemia with myelodysplasia-related changes, this hypothesis has never been systematically examined. We sought to investigate the clinicopathologic, cytogenetic, and molecular features of acute myeloid leukemia with erythroid predominance to subclassify cases as defined by the 2016 WHO. We retrospectively identified patients with $\geq 50 \%$ erythroid precursors and either $\geq 20 \%$ bone marrow blasts or $\geq 20 \%$ peripheral blood blasts at the time of initial diagnosis at seven major academic centers. Laboratory and clinical data were obtained. Patients were then reclassified according to 2016 WHO guidelines. A matched control group was also obtained. We identified 146 patients with acute myeloid leukemia with erythroid predominance $(62 \% \mathrm{M}$, average age: $62 \mathrm{y}$, range: 5-93y). Of these, 91 were acute myeloid leukemia with myelodysplasiarelated changes, $20(14 \%)$ were therapy-related myeloid neoplasm, $23(16 \%)$ acute myeloid leukemia, not otherwise specified, and ten acute myeloid leukemia with recurrent cytogenetic/molecular abnormalities. The bone marrow blast count ranged from $9-41 \%$. There was no difference in survival for patients with erythroid predominance compared to patients with acute myeloid leukemia without erythroid proliferations. In a multivariable analysis, cytogenetic risk was the only significant predictor of survival. We find a significantly lower rate of FLT3 and RAS pathway alterations in acute myeloid leukemia with erythroid predominance compared to controls. Our study is one of the first to apply the 2016 WHO guidelines for classification of acute myeloid leukemia. We find acute myeloid leukemia with erythroid predominance is a heterogeneous group and that erythroid richness has no impact on overall survival.
\end{abstract}

Robert P. Hasserjian and Attilio Orazi contributed equally to this work.

This is a Bone Marrow Pathology Working Group Study. Dr. Arber is currently in the Department of Pathology, University of Chicago.

$\square$ Elizabeth Margolskee

emm9030@med.cornell.edu

1 Department of Pathology and Laboratory Medicine, Weill Cornell Medical College/New York Presbyterian Hospital, New York, NY, USA

2 Department of Pathology and Laboratory Medicine, Hospital of the University of Pennsylvania, Philadelphia, PA, USA

3 Department of Hematopathology, The University of Texas M.D. Anderson Cancer Center, Houston, TX, USA

\section{Introduction}

The 2016 WHO revision introduced changes into the classification of acute myeloid leukemia cases with erythroid predominance, defined as $\geq 50 \%$ bone marrow erythroid cells. Previously, in WHO 2008, the blast percentage was

4 Department of Pathology, Stanford University Medical Center, Stanford, CA, USA

5 Department of Pathology, University of New Mexico Health Sciences Center, Albuquerque, NM, USA

6 Department of Pathology and Laboratory Medicine, Cleveland Clinic, Cleveland, OH, USA

7 Department of Pathology, Massachusetts General Hospital, Boston, MA, USA 
calculated using non-erythroid cells in the denominator and if blasts were $\geq 20 \%$ of non-erythroid cells, a diagnosis of acute erythroleukemia, erythroid/myeloid subtype, was rendered. In the 2016 revision, blast percentage is calculated using all nucleated cells in the denominator; thus, $\geq 20 \%$ blasts in bone marrow or peripheral blood are required for a diagnosis of acute myeloid leukemia, regardless of the presence or absence of erythroid predominance. The diagnosis of acute erythroleukemia, erythroid/myeloid subtype has been eliminated. This change was introduced because cases of acute erythroleukemia, erythroid/myeloid subtype in which blasts comprised $<20 \%$ of total cells display clinical, pathologic, and genetic features closer to myelodysplastic syndrome with excess blasts than to other acute myeloid leukemia cases [1].

Despite this change in classification, the clinical significance of erythroid proliferations in myeloid neoplasms remains unclear. In myelodysplastic syndrome with excess blasts, erythroid predominance has been associated with shorter survival in some studies, but not others $[1,2]$. However, these studies were limited to patients with $<20 \%$ blasts, which, according to WHO 2016 criteria, would be classified as myelodysplastic syndrome with excess blasts $[3,4]$. To our knowledge, erythroid predominance in acute myeloid leukemia has not been systematically examined. Although acute myeloid leukemia with erythroid predominance has historically been presumed to represent acute myeloid leukemia with myelodysplasia-related changes [5], this hypothesis has never been tested and the prognostic implications of erythroid predominance in acute myeloid leukemia according to the 2016 WHO definition remain unknown.

In this study, we identify a cohort of 146 acute myeloid leukemia with erythroid predominance classified according to the 2016 WHO Classification criteria. We find that, while the majority of cases represent acute myeloid leukemia with myelodysplasia-related changes, a proportion fall into other subtypes of acute myeloid leukemia. In this retrospective case-control study, we demonstrate that erythroid predominance in acute myeloid leukemia is most often associated with an intermediate cytogenetic risk profile and appears to have no effect on overall survival when compared to acute myeloid leukemia with $<50 \%$ erythroid cells. We also found erythroid predominant cases had a lower rate of FLT3 and KRAS/NRAS mutations than acute myeloid leukemia without erythroid predominance.

\section{Materials and methods}

We searched the pathology archives at seven large academic medical centers in the United States: Weill Cornell Medicine, Massachusetts General Hospital, Stanford University

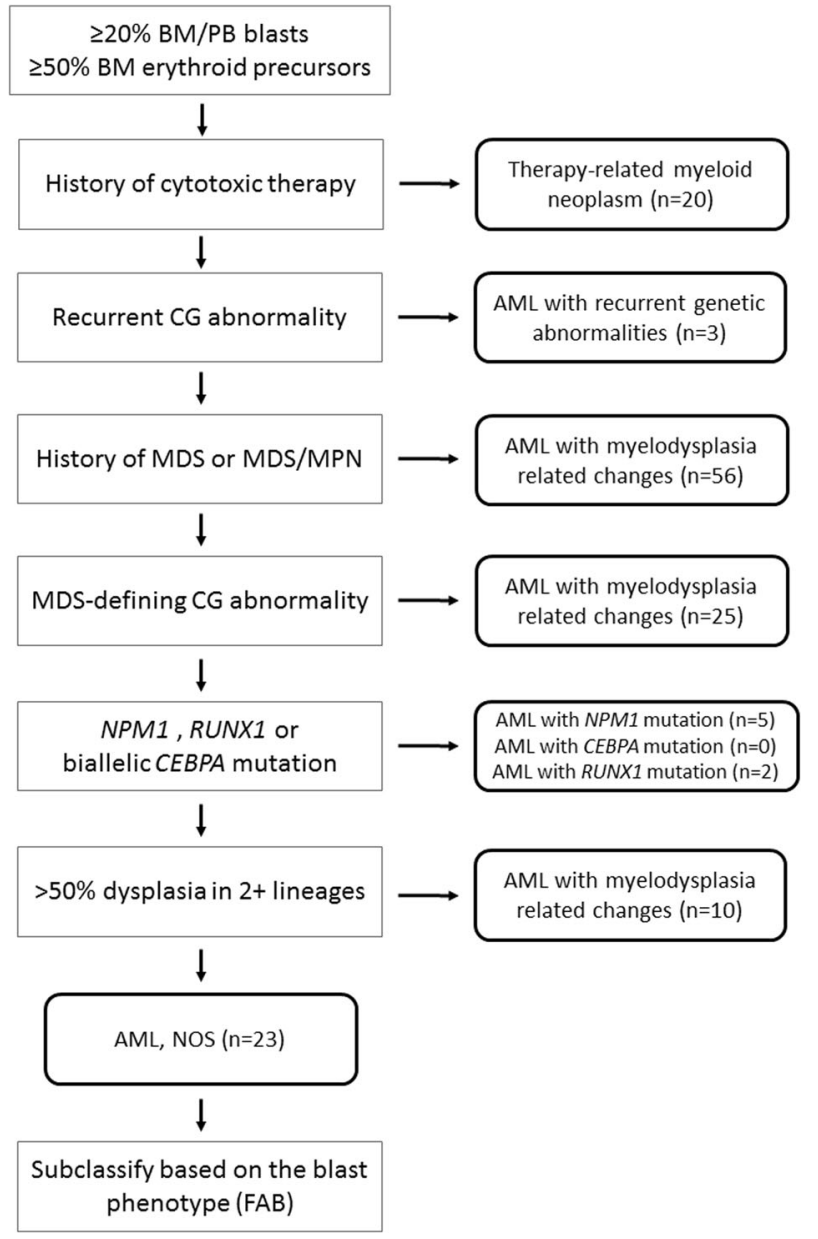

Fig. 1 Classification of cases, excluding two cases of blast phase myeloproliferative neoplasm

Medical Center, M.D. Anderson Cancer Center, Cleveland Clinic, Hospital of the University of Pennsylvania and the University of New Mexico for cases of acute myeloid leukemia newly diagnosed between 2008 and 2016 in which the bone marrow or peripheral blood blast count was $\geq 20 \%$ and the bone marrow erythroid cells were $\geq 50 \%$. Clinical information, laboratory data, bone marrow karyotype results and follow-up information were retrieved from the electronic medical records. Only limited treatment information was available for this retrospective study. These cases were then classified according to the 2016 WHO guidelines as acute myeloid leukemia with recurrent genetic abnormalities, acute myeloid leukemia with myelodysplasia-related changes, therapy related myeloid neoplasm, or acute myeloid leukemia, not otherwise specified, as appropriate (Fig. 1); acute myeloid leukemia, not otherwise specified were further subtyped based on the blast characteristics into the specific 2016 acute myeloid leukemia, not otherwise specified categories. Two control groups were collected. The first comprised an unbiased sample of 148 consecutively diagnosed cases of acute myeloid leukemia 
without erythroid predominance (i.e., bone marrow erythroid cells $<50 \%$; bone marrow or peripheral blood blasts $\geq 20 \%$ ) collected from a single institution used only as a comparator to determine incidence of acute myeloid leukemia subtypes when applying the 2016 WHO criteria. The second was a group of 155 cases of acute myeloid leukemia without erythroid predominance which was matched to the erythroid predominant cases according to the WHO subtypes of acute leukemia. This study was approved by the Institutional Review Boards of all participating institutions. This study was conducted in accordance with the Declaration of Helsinki.

\section{Pathology review}

Blood smears, bone marrow aspirate smears, and core biopsy specimens were reviewed by at least one author of this paper at each institution to confirm the diagnosis and to establish the 2016 WHO disease subtype. Peripheral blood blast counts were obtained by manual count of 200 cells. Bone marrow cellularity was estimated from the core biopsy. The myeloid:erythroid ratio was calculated from the aspirate counts. A differential count based on 500 cells or all available cells was performed on the bone marrow aspirate smears. Cytochemistry for myeloperoxidase and non-specific esterase was performed on bone marrow aspirate smears. Dysplastic changes had to be present in at least $50 \%$ of the cells in a lineage to consider the lineage dysplastic for the purposes of acute myeloid leukemia with myelodysplasia-related changes classification.

\section{Cytogenetics}

Conventional cytogenetic analysis was performed on Gbanded metaphase cells prepared from unstimulated bone marrow aspirate cultures using standard techniques. When possible, twenty metaphases were analyzed and the results reported using the International System for Human Cytogenetic Nomenclature. Karyotypes were stratified according to the United Kingdom Medical Research Council criteria for acute myeloid leukemia [6].

\section{Mutation studies}

Myeloid mutational studies by next generation sequencing were performed as part of the routine clinical workup in a subset of cases and controls at the original institution or at a commercial reference laboratory.

\section{Statistical analyses}

For continuous variables, data are reported as median and range; statistical significance was determined by unpaired $t$-test. For categorical and ordinal variables, data are reported as the number of patients if not specified. Statistical significance was assessed by Fisher's exact test or $\chi^{2}$ test, as appropriate, for categorical variables and by the Kruskal-Wallis test for ordinal variables. Overall survival was calculated from the day of diagnosis to the date of death or last follow-up. Distributions of overall survival were estimated by Kaplan-Meier curves. Multivariable analysis of survival was also performed using a Cox Proportionate Hazard model. The proportional hazard assumption was checked in each of the univariate Cox regression analyses. All $p$ values are two-tailed and considered significant when $<0.05$. Statistical analysis was performed using R software for Windows.

\section{Results}

A total of 146 cases of acute myeloid leukemia with erythroid predominance were identified. According to the 2016 WHO Classification, these were classified as 91 acute myeloid leukemia with myelodysplasia-related changes (62\%), 20 therapy-related myeloid neoplasm (14\%), 23 acute myeloid leukemia, not otherwise specified (16\%), ten acute myeloid leukemia with recurrent genetic abnormalities $(6.8 \%)$, and two cases $(1.3 \%)$ of blast phase of a prior myeloproliferative neoplasm. Within the acute myeloid leukemia, not otherwise specified group, the most common subtype was acute myeloid leukemia without maturation (FAB M1) $(n=9)$, followed by acute myeloid leukemia with maturation (FAB M2) $(n=4)$, acute myeloid leukemia with minimal differentiation (FAB M0) $(n=3)$, and acute myelomonocytic leukemia $(n=2)$. Subtype could not be determined in five cases because necessary studies were not done. Within the acute myeloid leukemia with recurrent genetic abnormalities group, five $(3 \%)$ were acute myeloid leukemia with NPMI mutation, two (1\%) were acute myeloid leukemia with inv(3), two (1\%) were acute myeloid leukemia with RUNXI mutation, and one $(1 \%)$ was acute myeloid leukemia with $t(6 ; 9)$. The incidence of acute myeloid leukemia with erythroid predominance among all acute myeloid leukemia was evaluated at two of the participating institutions, and was $1 \%(7 / 690)$ at Weill Cornell Medical College and 1.6\% (7/451) at Massachusetts General Hospital. Compared to an unbiased sample of 148 consecutively diagnosed cases of acute myeloid leukemia without erythroid predominance diagnosed over a similar time period, we observed a significant association between acute myeloid leukemia with erythroid predominance and acute myeloid leukemia with myelodysplasia-related changes $(p=0.0001)$. Conversely, the incidence of acute myeloid leukemia with recurrent genetic abnormalities was significantly lower in our acute myeloid leukemia with 
Table 1 Incidence of AML subtypes in AML with and without erythroid predominance

\begin{tabular}{llllll}
\hline & AML-EPa $(n)$ & $\%$ & Controls $(n)$ & $\%$ & $p$ \\
\hline AML-MRC & 91 & 62 & 48 & 32 & 0.0001 \\
t-AML & 20 & 14 & 25 & 17 & 0.69 \\
AML, NOS & 23 & 16 & 32 & 22 & 0.47 \\
AML-RGA & 10 & 7 & 43 & 29 & 0.0001 \\
\hline
\end{tabular}

${ }^{a}$ Excluding two cases of blast phase MPN

erythroid predominance group $(p=0.0001)$ : no cases of acute myeloid leukemia with $t(15 ; 17), t(8 ; 21)$, or inv [7] were identified in the acute myeloid leukemia with erythroid predominance group. There was no difference in the incidence of therapy related myeloid neoplasm and acute myeloid leukemia, not otherwise specified between the two groups (Table 1).

Clinical and pathologic features of the acute myeloid leukemia with erythroid predominance patients are summarized in Table 2. Representative photomicrographs of acute myeloid leukemia with erythroid predominance are presented in Fig. 2. The median age was 64 years (range 5-93). There was a notable male predominance (M:F ratio: 2.4). The median follow-up period was 1.5 years (range: 0-9 years). Data about erythropoietin treatment was available for a subset of patients. Erythropoietin therapy was used in 11/136 (13\%) patients with erythroid predominance prior to their diagnosis with acute myeloid leukemia and 6/ 11 (55\%) had erythropoietin levels above $20 \mathrm{mU} / \mathrm{mL}$. Among the individuals who were not treated with erythropoietin and with available erythropoietin levels, 31/73 (42\%) had levels above $20 \mathrm{mU} / \mathrm{mL}$, which was not significantly different from the erythropoietin-treated patients $(p=0.54)$. Significant dysplasia, defined as dyspoiesis affecting $>50 \%$ of cells at least one hematopoietic lineage, was seen in 53\% of cases. Dyserythropoiesis was most common (44\%), followed by dysmegakaryopoiesis (33.8\%) and dysgranulopoiesis (29.4\%). Flow cytometric data was available for a subset of patients. The blasts had a myeloid immunophenotype in all cases, with expression of CD34 in $108 / 134(81 \%)$, CD117 in 122/134 (91\%) and MPO in 54/ $72(75 \%)$. Rare cases $(4 / 126,3 \%)$ demonstrated expression of erythroid markers (CD71 and CD235a) on the myeloblasts. Megakaryocytic differentiation was not observed in any cases. Conventional karyotype showed intermediate and unfavorable risk in $59.6 \%$ and $40.4 \%$ of patients, respectively, according to the UKMRC scheme; favorable risk karyotype was not identified in any of the cases.

To assess the clinical significance of erythroid predominance in acute myeloid leukemia, we performed a case-control study using a second control cohort of 155 acute myeloid leukemia with $<50 \%$ bone marrow erythroid cells that excluded acute myeloid leukemia with recurrent
Table 2 Comparison of clinical, laboratory, and molecular data for AML-EP and AML without EP

\begin{tabular}{|c|c|c|c|}
\hline & $\begin{array}{l}\text { AML-EP }^{\mathrm{a}} \\
(N=134)\end{array}$ & $\begin{array}{l}\text { AML without } \\
\operatorname{EP}^{\mathrm{a}}(n=155)\end{array}$ & Significance \\
\hline Gender (M/F) & $103 / 43$ & $93 / 57$ & NS \\
\hline Age & 64.4 & 65.3 & NS \\
\hline AML-MRC & 91 & 98 & NS \\
\hline - Clinical history & 56 & 50 & NS \\
\hline - Karyotype & 25 & 42 & \\
\hline - Morphology only & 10 & 6 & \\
\hline T-AML & 20 & 25 & \\
\hline AML, NOS & 23 & 24 & \\
\hline BM blasts $(\%)$ & 24.8 & 51.2 & $p<0.0001$ \\
\hline BM erythroid cells (\%) & 55.6 & 14.2 & $p<0.0001$ \\
\hline PB WBC & 3.0 & 18.9 & $p<0.0001$ \\
\hline Hemoglobin (g/dL) & 9.0 & 9.1 & NS \\
\hline Platelets & 67.8 & 88.9 & NS \\
\hline PB Blasts (\%) & 21.7 & 29.8 & $p=0.002$ \\
\hline \multicolumn{4}{|l|}{ CG risk group (UKMRC) } \\
\hline Intermediate & $87(59 \%)$ & $73(48 \%)$ & $p=0.03$ \\
\hline Adverse & $59(41 \%)$ & $81(52 \%)$ & \\
\hline Received HSCT (\%) & $33(24 \%)$ & $49(32 \%)$ & NS \\
\hline \multicolumn{4}{|c|}{ Molecular data (mutation detected/cases tested) } \\
\hline$N P M 1^{\mathrm{b}}$ & $3 / 82(3.6 \%)$ & $8 / 108(7.4 \%)$ & NS \\
\hline FLT3 & $\begin{array}{l}3 / 101 \\
(2.9 \%)\end{array}$ & $11 / 107(10.3 \%)$ & $p=0.027$ \\
\hline TP53 & $\begin{array}{l}15 / 34 \\
(44 \%)\end{array}$ & $21 / 88(24 \%)$ & $p=0.045$ \\
\hline$N R A S / K R A S$ & $4 / 64(6.2 \%)$ & $16 / 41(39 \%)$ & $p=0.0001$ \\
\hline$I D H 1 / 2$ & $8 / 38(21 \%)$ & $12 / 35(34 \%)$ & NS \\
\hline$E Z H 2$ & $1 / 29(3 \%)$ & $4 / 30(13 \%)$ & NS \\
\hline$A S X L 1$ & $\begin{array}{l}3 / 26 \\
(11.5 \%)\end{array}$ & $8 / 33(24 \%)$ & NS \\
\hline
\end{tabular}

$A M L-E P$ AML with erythroid predominance, $A M L-M R C$ AML with myelodysplasia-related changes, $t-A M L$ therapy-related myeloid neoplasm, $B M$ bone marrow, $P B$ peripheral blood, $N S$ not significant, $H S C T$ hematopoietic stem cell transplant, $C G$ cytogenetic

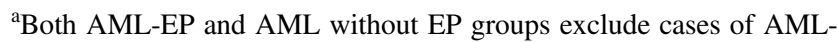
RGA and blast phase of MPN

${ }^{\mathrm{b}}$ Includes NPM1 mutated t-AML, AML-MRC by clinical history, and AML-MRC due to cytogenetic changes

genetic abnormalities or blast phase of myeloproliferative neoplasm cases, because these cases were few in the acute myeloid leukemia with erythroid predominance group. This control group was matched to the acute myeloid leukemia with erythroid predominance group proportionally for the number of acute myeloid leukemia with myelodysplasiarelated changes, therapy-related myeloid neoplasm, and acute myeloid leukemia, not otherwise specified cases. Comparing the acute myeloid leukemia with erythroid predominance cases to this second control group, there were 
Fig. 2 Representative images of acute myeloid leukemia with erythroid predominance. $\mathbf{a}, \mathbf{b}$ Bone marrow biopsy and aspirate smear showing acute myeloid leukemia, not otherwise specified with erythroid predominance. The marrow is hypercellular with expansion of erythroid precursors and myeloblasts. Only minimal dysplasia is present. c, $\mathbf{d}$ Bone marrow biopsy and aspirate smear showing acute myeloid leukemia with myelodysplasiarelated changes with erythroid predominance. The marrow is hypercellular with increased erythroid precursors and myeloblasts. Dyserythropoiesis and megakaryopoiesis (inset) are present
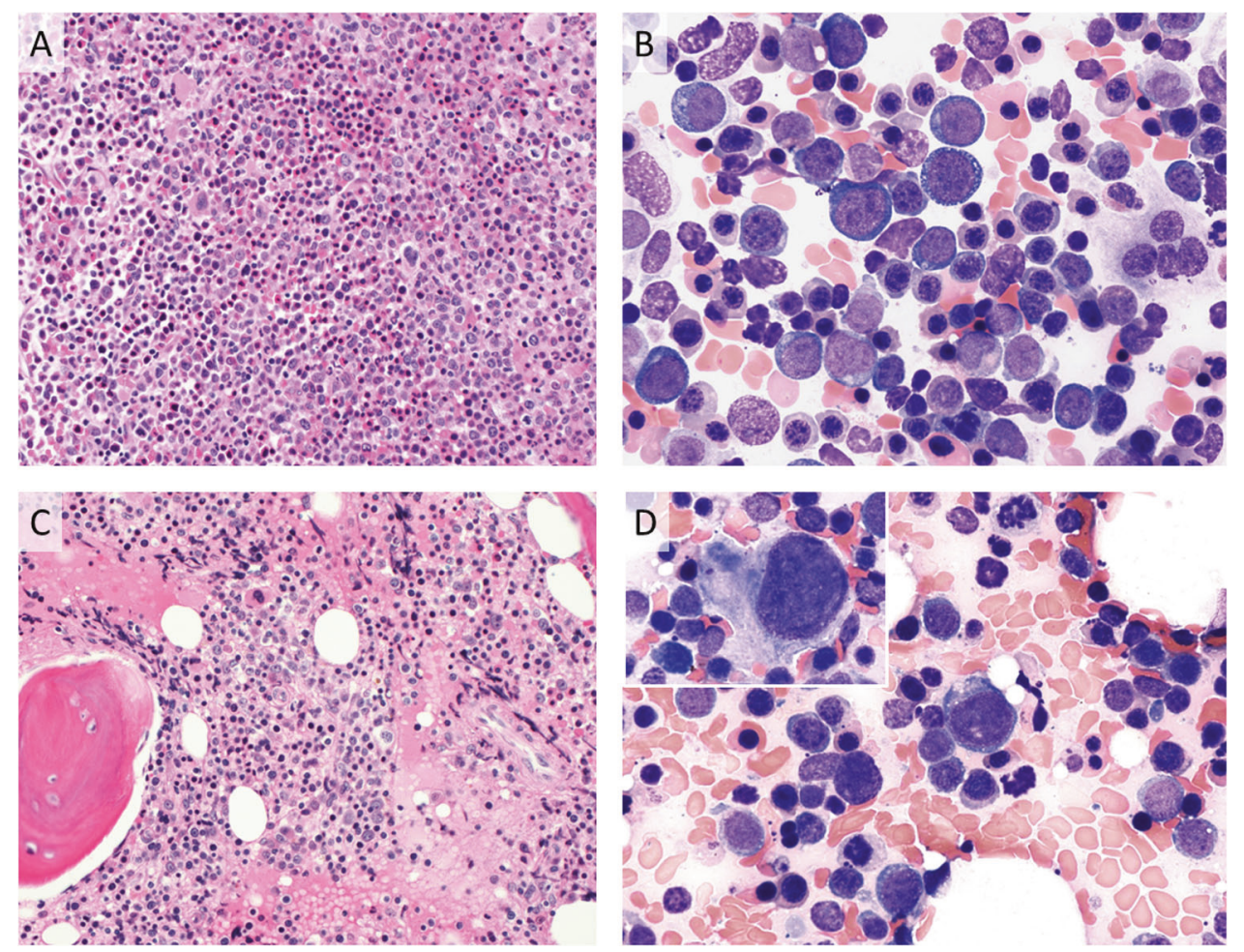

no significant differences in age, gender, or rate of hematopoietic stem cell transplantation between the two groups. As would be expected, cases of acute myeloid leukemia with erythroid predominance had lower bone marrow blast counts and a higher percentage of bone marrow erythroid cells than the control group ( $p<0.0001$ for both). Acute myeloid leukemia with erythroid predominance was associated with significantly lower peripheral blood leukocyte counts and lower levels of circulating blasts compared to acute myeloid leukemia without erythroid predominance ( $p$ $<0.0001$ and $p=0.002$, respectively). There was also a significantly lower proportion of acute myeloid leukemia with erythroid predominance cases harboring UKMRC adverse risk karyotypic abnormalities (41\% of acute myeloid leukemia with erythroid predominance vs. $52 \%$ of controls; $p=0.03$ ). In a Kaplan-Meier analysis, the median overall survival of acute myeloid leukemia with erythroid predominance patients was 260 days, nearly identical to that seen in the control acute myeloid leukemia patients (265 days). Within the acute myeloid leukemia with erythroid predominance cases, no significant differences in cytogenetic risk profile or overall survival were observed between cases with significant dyserythropoiesis and those without ( $p=0.07$ and $p=0.52$, respectively). In subgroup analyses within each 2016 WHO category (acute myeloid leukemia with myelodysplasia-related changes, therapyrelated myeloid neoplasm, and acute myeloid leukemia, not otherwise specified), there were no significant differences in survival observed between cases and controls. The median

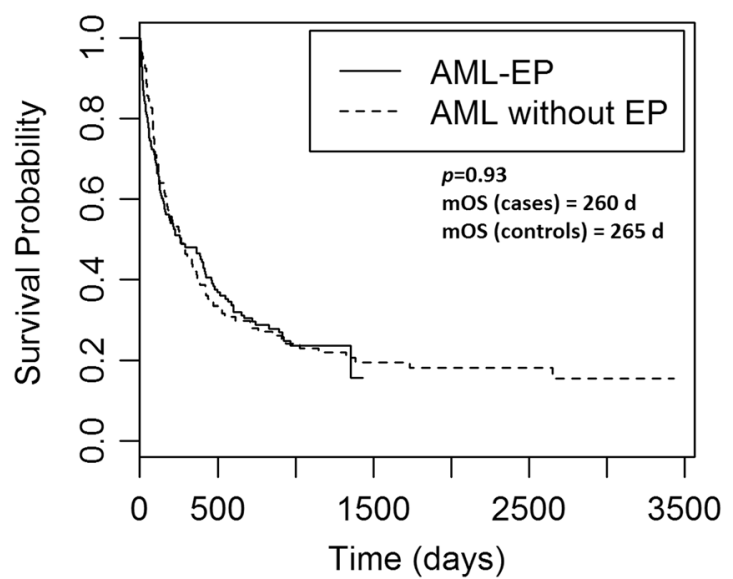

Fig. 3 Median overall survival for patients with and without erythroid predominance

overall survival for therapy-related myeloid neoplasm, acute myeloid leukemia with myelodysplasia-related changes, and acute myeloid leukemia, not otherwise specified with erythroid predominance was 150 days, 194 days, and 470 days, respectively. (Fig. 3) Thus, in this univariate analysis, erythroid predominance in acute myeloid leukemia had no impact on survival when the WHO 2016 subgroup was taken into account. Furthermore, using a Cox Proportional Hazards Model, the only variable associated with survival in acute myeloid leukemia with erythroid predominance and the control group was cytogenetic risk according to UKMRC ( $p=0.006)$ (Table 3). 
Table 3 Multivariable analysis of overall survival

\begin{tabular}{llll}
\hline & Hazard ratio & $95 \%$ CI & $p$ \\
\hline UKMRC cytogenetic risk category & 1.4 & $1.1-2$ & 0.006 \\
Erythroid predominance & 0.96 & $0.7-1.2$ & 0.8 \\
WHO Diagnosis (AML-MRC, AML- & 1.06 & $0.9-1.2$ & 0.36 \\
NOS, or t-AML) & & & \\
\hline
\end{tabular}

Mutation testing was performed on the majority of cases $(110 / 134,82 \%)$ and controls $(114 / 155,73 \%)$ at each referring center as part of the initial diagnostic evaluation (Table 2). There was no significant difference in the usage of molecular evaluation between cases and controls. Patients with acute myeloid leukemia with erythroid predominance had a significantly lower incidence of NRAS and $K R A S$ mutations compared to acute myeloid leukemia without erythroid predominance (combined NRAS/KRAS mutations 6.2 vs. 39\%, respectively; $p=0.0001$ ). The incidence of FLT3 mutations was also significantly lower in cases compared to controls (4.5\% and $10.3 \% ; p=0.027)$. Conversely, TP53 mutations were more common in cases compared to controls ( $44 \%$ vs $24 \% ; p=0.045$ ). There was no significant difference in the incidence of $I D H 1, I D H 2$, RUNX1, TET2, DNMT3A, and ASXL1 mutations between cases and controls (Table 2).

\section{Discussion}

In this retrospective study, we evaluated the clinical, morphologic, and genetic features of acute myeloid leukemia with erythroid predominance and compared them to acute myeloid leukemia without erythroid predominance. Applying the current WHO 2016 definition, erythroid predominance ( $\geq 50 \%$ bone marrow erythroid elements) is seen in only a small minority (1-1.6\%) of acute myeloid leukemia. We found that although most of these acute myeloid leukemia with erythroid predominance are classified as acute myeloid leukemia with myelodysplasia-related changes, a substantial proportion are classified as acute myeloid leukemia, not otherwise specified with smaller subsets classified as therapy related myeloid neoplasm or acute myeloid leukemia with recurrent genetic abnormalities. The presence of erythroid predominance made no impact on overall survival when compared to a matched control group of acute myeloid leukemia without erythroid predominance, with both groups in our study having a median overall survival of 260-265 days.

Erythroid predominance has historically been associated with acute myeloid leukemia arising from an antecedent myelodysplastic syndrome [5], but this assumption has never been systemically evaluated [8]. Our study confirms that acute myeloid leukemia with myelodysplasia-related changes is significantly overrepresented in cases of acute myeloid leukemia with erythroid predominance, validating its historic association with myelodysplastic syndrome. This overrepresentation of acute myeloid leukemia with myelodysplasia-related changes in acute myeloid leukemia with erythroid predominance is not likely due to patient age, which was similar in the erythroid-predominant and nonerythroid predominant acute myeloid leukemia cohorts: the median age in the control group was 61.0 years, compared to 64.5 in the acute myeloid leukemia with erythroid predominance group ( $p=0.06$; unpaired $t$-test).

We also note a lower proportion of acute myeloid leukemia with recurrent genetic abnormalities in our study group, which contained no cases of acute promyelocytic leukemia with $P M L-R A R A$ rearrangement, no leukemias with biallelic CEBPA mutations and relatively few with mutated NPM1. Although balanced translocations were seen in rare cases with erythroid predominance, the translocations associated with a favorable prognosis were entirely absent in this patient population. Interestingly, leukemias harboring these alterations characteristically have a morphology associated with myeloid differentiation, such as the abnormal eosinophils seen in acute myeloid leukemia with inv(3) [7]. The absence of these three recurrent genetic abnormalities from acute myeloid leukemia with erythroid predominance suggests that either these alterations occur in a hematopoietic cell more committed to myeloid differentiation, or that they impair differentiation of immature cells toward the erythroid lineage. Supporting these hypotheses, various studies from patients on acute promyelocytic leukemia confirm that the PML-RARA rearrangement is restricted to the myeloid lineage and is not observed in erythroid or lymphoid cells [9-11]. Studies evaluating lineage involvement in acute myeloid leukemia with $t(8 ; 21)$ have also shown absence of this chromosomal rearrangement from the erythroid compartment [12]. The findings in our acute myeloid leukemia with erythroid predominance cohort confirm that erythroid expansion is infrequently if ever seen in conjunction with these favorable risk cytogenetic abnormalities.

In contrast, we observed two cases of acute myeloid leukemia with erythroid predominance with inv(3) and one with $t(6 ; 9)$, two recurrent cytogenetic abnormalities which are associated with morphologic dysplasia and an unfavorable prognosis; multilineage dysplasia was seen in all of these three acute myeloid leukemia with recurrent genetic abnormalities cases in our cohort. Similarly, therapy-related myeloid neoplasia is commonly associated with multilineage dysplasia and bone marrow failure, especially in patients with exposure to alkylating treatment or radiotherapy. We observed that 14/20 (70\%) cases of therapy related myeloid neoplasm with erythroid predominance had 
multilineage dysplasia. Thus, we find that erythroid predominance is highly associated with dysplastic features morphologically.

We found NPM1 mutation in $5.4 \%$ of acute myeloid leukemia with erythroid predominance, a much lower proportion than is observed in acute myeloid leukemia overall, where $20-30 \%$ of acute myeloid leukemia are expected to harbor NPM1 mutation [13].. Moreover, most of these NPM1-mutated cases were classified as acute myeloid leukemia with myelodysplasia-related changes due to the karyotype or therapy-related myeloid neoplasm; only $3 \%$ of the acute myeloid leukemia with erythroid predominance cases fulfilled WHO criteria for acute myeloid leukemia with mutated NPM1. Co-occurrence of NPM1 and FLT3 mutation was seen in three patients. Although the sample size was relatively small $(n=8)$, we found a trend towards improved survival for patients with NPMI mutation compared to acute myeloid leukemia with erythroid predominance lacking NPM1 mutation (median overall survival not reached vs 10 months, $p=0.053$; data not shown), similar to other studies of NPMI mutation in erythroid rich acute myeloid leukemia [4]. Similar to the prior study, our results suggest that among acute myeloid leukemia with erythroid predominance, the presence of NPMI mutation is associated with favorable prognosis, supporting the inclusion of these cases with NPM1 mutation within the category of acute myeloid leukemia with mutated NPM1 in the 2016 WHO Classification; however, larger studies are needed to confirm this finding.

We found that the cytogenetic risk group (using UKMRC criteria) [6] was most strongly correlated with survival in our cohort, while the presence or absence of significant $(\geq 50 \%)$ dyserythropoiesis had no impact on survival. This finding confirms several previous studies of erythroid predominance in acute myeloid leukemia, which also demonstrated a pivotal role for cytogenetics in providing prognostic information for patients with high-grade myeloid neoplasms with erythroid predominance [3, 7, 14]. In a multivariable model, no other covariates were significant predictors of outcome, including the presence or absence of $\geq 50 \%$ bone marrow erythroid cells. This is in contrast to a prior study, which demonstrated longer overall survival in myeloid leukemia with erythroid predominance in comparison to those without [3]. However, this study relied on 2008 WHO criteria for acute myeloid leukemia, which enumerated blasts as a percentage of non-erythroid cells and thus included cases which currently would be classified as myelodysplastic syndrome with excess blasts. To our knowledge, our study is the first to apply the updated diagnostic criteria for acute myeloid leukemia to evaluate the clinical significance of erythroid predominance. We find that when the $20 \%$ myeloblast threshold is applied uniformly to assign a diagnosis of acute myeloid leukemia, erythroid proliferation has no impact on outcome, supporting the elimination of the acute erythroid leukemia (erythroid/myeloid) category from the 2016 WHO Classification. Like other acute myeloid leukemia cases, the prognosis of leukemias with erythroid predominance is mainly driven by karyotype risk and also likely influenced by the mutational profile.

Recent advances in molecular biology, particularly following the introduction of next generation sequencing methodology, have allowed assessment of mutations as part of the routine diagnostic work-up of acute myeloid leukemia patients. In our cases of acute myeloid leukemia with erythroid predominance, FLT3 alterations were found in $2.9 \%$, similar to that reported by Zuo et al. and Grossmann et al. [3, 4]. The incidence of KRAS and NRAS mutations was also lower in acute myeloid leukemia with erythroid predominance compared to leukemia without erythroid predominance, as reported in prior studies [3]. These collective findings suggest that RAS pathway mutations are much less common in erythroid predominant acute myeloid leukemia than in acute myeloid leukemia without erythroid predominance. Mutations in TP53 were seen in 44\% of cases, similar to previous reports which range from $35-43.5 \%$ [4, 15]. However, these comparisons with prior studies evaluating the molecular landscape of myeloid neoplasia with erythroid proliferations must be interpreted with caution as these studies used the 2008 WHO criteria for diagnosis and include a large number of cases with $<20 \%$ blasts, which by 2016 criteria are classified as myelodysplastic syndrome with excess blasts rather than acute myeloid leukemia.

The biology underlying erythroid proliferations in acute myeloid leukemia remains unclear. Expanded erythropoiesis may be a reactive phenomenon driven by erythropoietin in patients with marked anemia, a concomitant metabolic deficiency, a paraneoplastic phenomenon due to secretion of soluble factors by myeloblasts that promote increased erythropoiesis, or erythroid differentiation of the neoplastic clone. The latter hypothesis may be supported by the observation of frequent erythroid lineage dysplasia in our cohort (43\% of the cases). In view of the clinical heterogeneity of the cases in our study, the pathogenesis of these erythroid proliferations may be variable as well. Future studies may be helpful in addressing these possibilities.

In summary, we present a comprehensive characterization of acute myeloid leukemia with erythroid predominance based on current 2016 WHO guidelines requiring at least $20 \%$ bone marrow blasts. Our study is the first to apply the new classification system to this challenging subset of acute myeloid leukemia patients. We find that acute myeloid leukemia with erythroid proliferation is a heterogeneous group that frequently, but not exclusively, includes acute myeloid leukemia with myelodysplasia- 
related changes and therapy-related cases, shows a relatively low incidence of $R A S$ pathway mutations and has a high incidence of TP53 mutations. The morphologic finding of increased erythroid cells did not have prognostic relevance in our study; rather, we confirm that, like nonerythroid predominant acute myeloid leukemia, the karyotype risk is the most important factor in providing prognostic information for these patients.

\section{Compliance with ethical standards}

Conflict of interest The authors declare that they have no conflict of interest.

\section{References}

1. Wang SA, Patel KP, Pozdnyakova O, et al. Acute erythroid leukemia with $<20 \%$ bone marrow blasts is clinically and biologically similar to myelodysplastic syndrome with excess blasts. Mod Pathol. 2016;29:1221-31.

2. Calvo X, Arenillas L, Luno E, et al. Erythroleukemia shares biological features and outcome with myelodysplastic syndromes with excess blasts: a rationale for its inclusion into future classifications of myelodysplastic syndromes. Mod Pathol. 2016;29:1541-51.

3. Zuo Z, Medeiros LJ, Chen Z, et al. Acute myeloid leukemia (AML) with erythroid predominance exhibits clinical and molecular characteristics that differ from other types of AML. PLoS One. 2012;7:e41485.

4. Grossmann V, Bacher U, Haferlach C, et al. Acute erythroid leukemia (AEL) can be separated into distinct prognostic subsets based on cytogenetic and molecular genetic characteristics. Leukemia. 2013;27:1940-3.

5. Arber DA, Orazi A, Hasserjian R, et al. The2016 revision to the World Health Organization classification of myeloid neoplasms and acute leukemia. Blood. 2016;127:2391-405.
6. Grimwade D, Hills RK, Moorman AV, et al. Refinement of cytogenetic classification in acute myeloid leukemia: determination of prognostic significance of rare recurring chromosomal abnormalities among 5876 younger adult patients treated in the United Kingdom Medical Research Council trials. Blood. 2010;116:354-65.

7. Hasserjian RP, Zuo Z, Garcia C, et al. Acute erythroid leukemia: a reassessment using criteria refined in the 2008 WHO classification. Blood. 2010;115:1985-92.

8. Dameshek W. The DiGuglielmo syndrome revisited. Blood. 1969;34:567-72.

9. Knuutila S, Teerenhovi L, Larramendy ML, et al. Cell lineage involvement of recurrent chromosomal abnormalities in hematologic neoplasms. Genes Chromosom Cancer. 1994;10:95-102.

10. Chang H, Qi XY, Sutherland DR. Identification of cell lineages involved by $\mathrm{t}(15 ; 17)$ in acute promyelocytic leukemia by combined fluorescence activated cell sorting and FISH. Cancer Genet Cytogenet. 2005;158:43-8.

11. Haferlach $T$, Loffler $H$, Nickenig $C$, et al. Cell lineage specific involvement in acute promyelocytic leukaemia (APL) using a combination of May-Grunwald-Giemsa staining and fluorescence in situ hybridization techniques for the detection of the translocation $\mathrm{t}(15 ; 17)(\mathrm{q} 22 ; \mathrm{q} 12)$. Br J Haematol. 1998;103:93-9.

12. van Lom K, Hagemeijer A, Vandekerckhove F, et al. Clonality analysis of hematopoietic cell lineages in acute myeloid leukemia and translocation $(8 ; 21)$ : only myeloid cells are part of the malignant clone. Leukemia. 1997;11:202-5.

13. Falini B, Mecucci C, Tiacci E, et al. Cytoplasmic nucleophosmin in acute myelogenous leukemia with a normal karyotype. N Engl J Med. 2005;352:254-66.

14. Bacher U, Haferlach C, Alpermann T, et al. Comparison of genetic and clinical aspects in patients with acute myeloid leukemia and myelodysplastic syndromes all with more than $50 \%$ of bone marrow erythropoietic cells. Haematologica. 2011;96: 1284-92.

15. Cervera N, Carbuccia N, Garnier S, et al. Molecular characterization of acute erythroid leukemia (M6-AML) using targeted next-generation sequencing. Leukemia. 2016;30:966-70. 